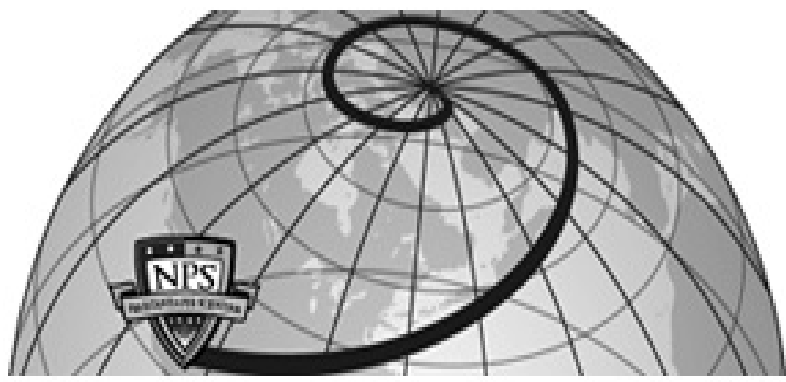

Calhoun: The NPS Institutional Archive DSpace Repository

\title{
Normal Forms and Bifurcations of Control Systems
}

Chang, D.-E.; Kang, W.; Krener, A.J.

https://hdl.handle.net/10945/40355

This publication is a work of the U.S. Government as defined in Title 17, United States Code, Section 101. Copyright protection is not available for this work in the United States.

Downloaded from NPS Archive: Calhoun

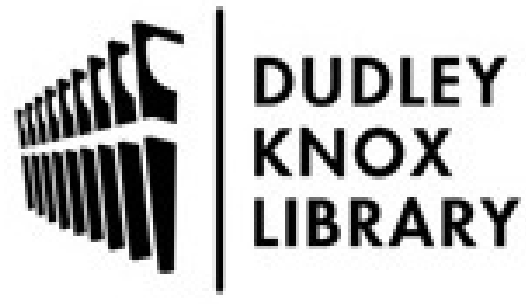

http://www.nps.edu/library
Calhoun is the Naval Postgraduate School's public access digital repository for research materials and institutional publications created by the NPS community. Calhoun is named for Professor of Mathematics Guy K. Calhoun, NPS's first appointed -- and published -- scholarly author.

Dudley Knox Library / Naval Postgraduate School 411 Dyer Road / 1 University Circle Monterey, California USA 93943 


\title{
Normal Forms and Bifurcations of Control Systems
}

\author{
D.-E. Chang \\ Control and Dynamical Systems, California Institute of Technology, Pasadena, CA 91125 \\ W. Kang \\ Department of Mathematics, Naval Postgraduate School, Monterey, CA 93943 \\ and \\ A. J. Krener ${ }^{1}$ \\ Department of Mathematics, University of California, Davis, CA 95616-8633
}

\begin{abstract}
We present the quadratic and cubic normal forms of a nonlinear control system around an equilibrium point. These are the normal forms under change of state coordinates and invertible state feedback. The system need not be linearly controllable. A control bifurcation of a nonlinear system occurs when its linear approximation loses stabilizability. We study some important control bifurcations, the analogues of the classical fold, transcritical and Hopf bifurcations.
\end{abstract}

Keywords: Normal Form, Control Bifurcation, Fold Control Bifurcation, Transcritical Control Bifurcation, Hopf Control Bifurcation.

\section{Introduction}

The theory of normal forms and bifurcations of dynamical systems is well-known [8]. One considers a smooth vector field

$$
\dot{x}=f(x, \mu)
$$

depending on a parameter $\mu$. The equilibria of the vector field are those $x_{e}, \mu_{e}$ such that $f\left(x_{e}, \mu_{e}\right)=0$. Perhaps the most important property of an equilibrium is its stability. In the first approximation this is determined by the stability of its linear approximation around $x_{e}, \mu_{e}$,

$$
\dot{\delta x}=\frac{\partial f}{\partial x}\left(x_{e}, \mu_{e}\right) \delta x .
$$

If all the eigenvalues of $\frac{\partial f}{\partial x}\left(x_{e}, \mu_{e}\right)$ lie in the open left half plane then the system (1.1) is locally asymptotically stable around the $x_{e}, \mu_{e}$. If one or more eigenvalues lie in the open right half plane then the system (1.1) is unstable. If all the eigenvalues lie in the closed

\footnotetext{
${ }^{1}$ Corresponding author. Research supported in part by AFOSR-49620-95-1-0409 and by NSF 9970998. To be presented at the IEEE CDC 2000, Sydney.
}

left half plane but some are on the imaginary axis then the first approximation is not decisive, the system (1.1) may be locally asymptotically stable or unstable, depending on higher degree terms.

The character of the equilibria can change at a critical value of the parameter, perhaps two branches of equilibria cross or a branch looses or gains stability. Such a state and parameter is called a bifurcation point of the parametrized vector field. A local bifurcation takes place at a parameter value where the system loses structural stability with respect to parameter variations, i.e. the phase portrait around the equilibrium at the critical parameter value is not locally topologically conjugate to the phase portraits around the equilibria at nearby parameter values. If the local linearizations at two equilibria have no poles on the imaginary axis and the same numbers of strictly stable and strictly unstable poles then the local phase portraits are topologically conjugate. Therefore a bifurcation is characterized mathematically by one or more eigenvalues of the linearized system crossing the imaginary axis. We restrict our discussion to local bifurcations which we refer to as bifurcations.

A standard approach to analyzing the behaviour of the parametrized ODE (1.1) around a bifurcation point is to treat the parameter as an additional state variable with dynamics $\dot{\mu}=0$ and to compute the center manifold of the extended dynamics through the bifurcation point and the dynamics restricted to this manifold [8]. The center manifold is an invariant manifold of the differential equation which is tangent at the bifurcation point to the eigenspace of the neutrally stable eigenvalues. In practice, one does not compute the center manifold and its dynamics exactly, in most cases of interest, an approximation of degree two or three suffices. If the other eigenvalues are in the open left half plane, then this part of the dynamics is locally asymptotically stable and therefore can be neglected in a local stability analysis around the bifurcation point. The bifurcation point will be locally asymptotically stable for the complete dynamics iff the dynamics on the center 
manifold is locally asymptotically stable. Of course, at some nearby equilibria the dynamics may be unstable.

The next step is to compute the Poincaré normal form of the center manifold dynamics. From its normal form the bifurcation is recognized and understood. Familiar examples are the fold (or saddle node), the transcritical and the Hopf bifurcations. The first two of these depend on the normal form of degree two and the last one depends on the normal form of degree three. The fold and Hopf bifurcations are the only ones that are generic and of codimension 1, i. e., depend on a single parameter, so these are the most important.

The study of bifurcations of differential equations with control was initiated by Abed and Fu, [1], [2]. They considered systems where the parameter is distinct from the control

$$
\dot{x}=f(x, u, \mu)
$$

They assumed that the uncontrolled system $u=0$ undergoes a bifurcation at a critical value of the parameter $\mu_{0}$ and they studied the stabilizability of the system by quadratic and cubic feedbacks.

Kang, [5] studied the degree two normal forms and bifurcations of control systems with (1.3) and without a parameter (1.4). A control system does not need a parameter to bifurcate, the control can play the same role. The equilibria of a controlled differential equation,

$$
\dot{x}=f(x, u)
$$

are those values of $x_{e}, u_{e}$ such that $f\left(x_{e}, u_{e}\right)=0$. The rquilibria are conveniently parametrized by $u$ or one of the state varaibles. Two key facts differentiate bifurcations of a control system (1.4) from that of a parametrized system (1.1). The first is that for the latter the structural stability of the equilibria is the crucial issue but for the former the stabilizability by state feedback is the crucial issue. A control system (1.4) is linearly controllable (linearly stabilizable) at $x_{c}, u_{e}$ if the local linear approximation

$$
\dot{\delta x}=\frac{\partial f}{\partial x}\left(x_{e}, u_{e}\right) \delta x+\frac{\partial f}{\partial u}\left(x_{e}, u_{e}\right) \delta u
$$

is controllable (stabilizable). If the linear approximation is stabilizable, then the nonlinear system is locally stabilizable. If the linear approximation is not stabilizable, then the nonlinear system may or may not be locally stabilizable, depending on higher degree terms. A control bifurcation of (1.4) takes place at an equilibrium where the linear approximation loses stabilizability. Notice that this is different from the bifurcation of a parametrized system (1.1) which take place at an equilibrium where there is a loss of structural stability with respect to parameter variations. Frequently this loss of structural stability is caused by a loss of linear stability, one or more eigevalues of (1.2) crossing the imaginary axis. To empahsize this distinction we shall refer to the latter as a classical bifurcation.

The other difference between control and classical bifurcations is that when bringing the control system into normal form, a different group of transformations is used. For classical bifurcations, we use parameter dependent change of state coordinates and change of parameter coordinates but for control bifurcations we use change of state coordinates and state dependent change of control coordinates (invertible state feedback) to simplify the dynamics.

After reviewing Kang's work on the normal forms of degree two control systems, we extend it to degree three normal forms. Similar results for discrete time systems can be found in [3], [7]. Kang studied the control theoretic analogue of the transcritical bifurcation. We extend this to a study of the control theoretic analogues of the fold and the Hopf bifurcations. We will study the stabilizability of these around the bifurcation point. We will also discuss the parametrized stabilizability of the parametrized family to nearby equilibria by a parametrized control law. Because of space limitations, the proofs will appear elsewhere [6].

\section{Normal Forms}

Consider a smooth $\left(C^{4}\right)$ control system (1.4) where $x$ is $n$ dimensional, $u$ is one dimensional and $f(0,0)=0$. It is well known that by linear change of state coordinates and linear state feedback, the system can be brought to the form

$$
\begin{aligned}
{\left[\begin{array}{c}
\dot{x}_{1} \\
\dot{x}_{2}
\end{array}\right]=} & {\left[\begin{array}{cc}
A_{1} & 0 \\
0 & A_{2}
\end{array}\right]\left[\begin{array}{c}
x_{1} \\
x_{2}
\end{array}\right]+\left[\begin{array}{c}
0 \\
B_{2}
\end{array}\right] u } \\
& +\left[\begin{array}{c}
f_{1}^{[2]}\left(x_{1}, x_{2}, u\right) \\
f_{2}^{[2]}\left(x_{1}, x_{2}, u\right)
\end{array}\right]+\left[\begin{array}{c}
f_{1}^{[3]}\left(x_{1}, x_{2}, u\right) \\
f_{2}^{[3]}\left(x_{1}, x_{2}, u\right)
\end{array}\right] \\
& +O\left(x_{1}, x_{2}, u\right)^{4}
\end{aligned}
$$

where $x_{1}, x_{2}$ are $n_{1}, n_{2}$ dimensional, $n_{1}+n_{2}=n, A_{1}$ is in Jordan form, $A_{2}, B_{2}$ are in controller (Brunovsky) form and $f_{i}^{[d]}\left(x_{1}, x_{2}, u\right)$ is a vector field which is a homogeneous polynomial of degree $d$ in its arguments. The linear change of coordinates that brings $A_{1}$ to Jordan form may be complex.

A pair $A_{2}, B_{2}$ is in controller form if

$$
A_{2}=\left[\begin{array}{ccccc}
0 & 1 & 0 & \ldots & 0 \\
0 & 0 & 1 & \ldots & 0 \\
& & \ddots & \ddots & \\
0 & 0 & 0 & \ldots & 1 \\
0 & 0 & 0 & \ldots & 0
\end{array}\right] \quad B_{2}=\left[\begin{array}{c}
0 \\
0 \\
\vdots \\
0 \\
1
\end{array}\right]
$$


The following theorem is a slight extension of Kang [5].

Theorem 2.1 There exist a quadratic change of coordinates and a quadratic feedback

$$
\begin{aligned}
{\left[\begin{array}{l}
z_{1} \\
z_{2}
\end{array}\right] } & =\left[\begin{array}{l}
x_{1} \\
x_{2}
\end{array}\right]-\left[\begin{array}{c}
\phi_{1}^{[2]}\left(x_{1}, x_{2}\right) \\
\phi_{2}^{[2]}\left(x_{1}, x_{2}\right)
\end{array}\right] \\
v & =u-\alpha_{1}^{[2]}\left(x_{1}, x_{2}, u\right)
\end{aligned}
$$

which transforms the system (2.5) into the quadratic normal form

$$
\begin{aligned}
{\left[\begin{array}{c}
\dot{z}_{1} \\
\dot{z}_{2}
\end{array}\right]=} & {\left[\begin{array}{cc}
A_{1} & 0 \\
0 & A_{2}
\end{array}\right]\left[\begin{array}{c}
z_{1} \\
z_{2}
\end{array}\right]+\left[\begin{array}{c}
0 \\
B_{2}
\end{array}\right] v } \\
& +\left[\begin{array}{c}
\tilde{f}_{1}^{[2 ; 0]}+\tilde{f}_{1}^{[1 ; 1]}+\tilde{f}_{1}^{[0 ; 2]} \\
0+0+\tilde{f}_{2}^{[0 ; 2]}
\end{array}\right] \\
& +\left[\begin{array}{c}
\tilde{f}_{1}^{[3]} \\
\tilde{f}_{1}^{[3]}
\end{array}\right]+O\left(z_{1}, z_{2}, v\right)^{4}
\end{aligned}
$$

where $\tilde{f}_{i}^{\left[d_{1} ; d_{2}\right]}=\tilde{f}_{i}^{\left[d_{1} ; d_{2}\right]}\left(z_{1} ; z_{2}, v\right)$ denotes a polynomial vector field homogeneous of degree $d_{1}$ in $z_{1}$ and of degree $d_{2}$ in $z_{2}, v$. The vector field $\tilde{f}_{1}^{[2 ; 0]}$ is in the quadratic normal form of Poincaré [8],

$$
\tilde{f}_{1}^{[2 ; 0]}=\sum_{\lambda_{i}=\lambda_{j}+\lambda_{k}} \beta_{i}^{j k} \mathbf{e}_{1}^{i} z_{1, j} z_{1, k}
$$

where $\mathbf{e}_{1}^{i}$ is the $i^{\text {th }}$ unit vector in $z_{r}$ space and $z_{r, i}$ is the $i^{\text {th }}$ component of $z_{r}$. The other vector fields are as follows.

$$
\begin{aligned}
\tilde{f}_{1}^{[1 ; 1]} & =\sum_{i=1}^{n_{1}} \sum_{j=1}^{n_{1}} \gamma_{i}^{j 1} \mathbf{e}_{1}^{i} z_{1, j} z_{2,1} \\
\tilde{f}_{1}^{[0 ; 2]} & =\sum_{i=1}^{n_{1}} \sum_{j=1}^{n_{2}+1} \delta_{i}^{j j} \mathbf{e}_{1}^{i} z_{2, j}^{2} \\
\tilde{f}_{2}^{[0 ; 2]} & =\sum_{i=1}^{n_{2}-1} \sum_{j=i+2}^{n_{2}+1} \zeta_{i}^{j j} \mathbf{e}_{2}^{i} z_{2, j}^{2}
\end{aligned}
$$

where for notational convenience we have defined $z_{2, n_{2}+1}=v$. The vector field, $f_{2}^{[0 ; 2]}$, is in quadratic controller form [4].

The following is theorem is new.

Theorem 2.2 There exist a quadratic and cubic change of coordinates and a quadratic and cubic feedback

$$
\begin{aligned}
{\left[\begin{array}{c}
\tilde{z}_{1} \\
\tilde{z}_{2}
\end{array}\right] } & =\left[\begin{array}{l}
x_{1} \\
x_{2}
\end{array}\right]-\left[\begin{array}{c}
\phi_{1}^{[2]}\left(x_{1}, x_{2}\right) \\
\phi_{2}^{[2]}\left(x_{1}, x_{2}\right)
\end{array}\right]-\left[\begin{array}{l}
\phi_{1}^{[3]}\left(x_{1}, x_{2}\right) \\
\phi_{2}^{[3]}\left(x_{1}, x_{2}\right)
\end{array}\right] \\
v & =u-\alpha_{1}^{[2]}\left(x_{1}, x_{2}, u\right)-\alpha_{1}^{[3]}\left(x_{1}, x_{2}, u\right)
\end{aligned}
$$

which transforms the system (2.5) into the cubic normal form

$$
\begin{aligned}
& {\left[\begin{array}{c}
\dot{z}_{1} \\
\dot{z}_{2}
\end{array}\right]=\left[\begin{array}{cc}
A_{1} & 0 \\
0 & A_{2}
\end{array}\right]\left[\begin{array}{l}
z_{1} \\
z_{2}
\end{array}\right]+\left[\begin{array}{c}
0 \\
B_{2}
\end{array}\right] v} \\
& +\left[\begin{array}{ccccc}
\tilde{f}_{1}^{[2 ; 0]} & + & \tilde{f}_{1}^{[1 ; 1]} & + & \tilde{f}_{1}^{[0 ; 2]} \\
0 & + & 0 & + & \tilde{f}_{2}^{[0 ; 2]}
\end{array}\right] \\
& +\left[\begin{array}{ccccccc}
\tilde{f}_{1}^{[3 ; 0]} & + & \tilde{f}_{1}^{[2 ; 1]} & + & \tilde{f}_{1}^{[1 ; 2]} & + & \tilde{f}_{1}^{[0 ; 3]} \\
0 & + & 0 & + & \tilde{f}_{2}^{[2 ; 1]} & + & \tilde{f}_{2}^{[0 ; 3]}
\end{array}\right] \\
& +O\left(z_{1}, z_{2}, v\right)^{4}
\end{aligned}
$$

where the quadratic part is as in the previous theorem. Furthermore $\tilde{f}_{1}^{[3 ; 0]}$ is in the cubic normal form of Poincaré [8],

$$
\tilde{f}_{1}^{[3 ; 0]}=\sum_{\lambda_{i}=\lambda_{j}+\lambda_{k}+\lambda_{l}} \beta_{i}^{j k l} \mathbf{e}_{1}^{i} z_{1, j} z_{1, k} z_{1, l}
$$

The other cubic vector fields are as follows.

$$
\begin{aligned}
\tilde{f}_{1}^{[2 ; 1]} & =\sum_{i=1}^{n_{1}} \sum_{j=1}^{n_{1}} \sum_{k=j}^{n_{1}} \gamma_{i}^{j k 1} \mathbf{e}_{1}^{i} z_{1, j} z_{1, k} z_{2,1} \\
\tilde{f}_{1}^{[1 ; 2]} & =\sum_{i=1}^{n_{1}} \sum_{j=1}^{n_{1}} \sum_{k=1}^{n_{2}+1} \delta_{i}^{j k k} \mathbf{e}_{1}^{i} z_{1, j} z_{2, k}^{2} \\
\tilde{f}_{1}^{[0 ; 3]} & =\sum_{i=1}^{n_{1}} \sum_{j=1}^{n_{2}} \sum_{k=j}^{n_{2}+1} \epsilon_{i}^{j k k} \mathbf{e}_{1}^{i} z_{2, j} z_{2, k}^{2} \\
\tilde{f}_{2}^{[2 ; 1]} & =\sum_{i=1}^{n_{2}-1} \sum_{j=1}^{n_{1}} \sum_{k=i+2}^{n_{2}+1} \zeta_{i}^{j k k} \mathbf{e}_{2}^{i} z_{1, j} z_{2, k}^{2} \\
f_{2}^{[0 ; 3]} & =\sum_{i=1}^{n_{2}-1} \sum_{k=i+2}^{n_{2}+1} \sum_{j=1}^{k} \eta_{i}^{j k k} \mathbf{e}_{2}^{i} z_{2, j} z_{2, k}^{2} .
\end{aligned}
$$

These theorems hold even when some of the eigenvalues of $A_{1}$ are not real. The linear change of coordinates that diagonalizes $A_{1}$ is complex and the corresponding coefficients in the normal forms are complex. The real dimension of the parameter space is unchanged as the complex coordinates come in conjugate pairs hence some coefficients are required to be complex conjugates of each other.

\section{Control Bifurcations}

The constants in the above normal forms $\lambda_{i}, \beta_{i}^{j k}, \ldots, \eta_{i}^{j k k}$ are called moduli and there are a lot more than are needed to understand the simplest types of bifurcations that are possible. Recall that in the bifurcation theory of a parametrized system of ODEs, the interesting part of the dynamics is that restricted to the center manifold. This leads to a great reduction in the dimension of the ODE that 
nust be studied. A similar fact holds true when studying control bifurcations. In most applications one will ultimately use state feedback in an attempt to stabilize the system so the coordinates that are linearly stabilizable can be ignored to a large extent. If there are modes which are neutrally stable and are not linearly stabilizable, then the particular choice of feedback will influence the shape of center manifold of the closed loop system and the dynamics thereon. It might be possible to achieve asymptotically stable center manifold dynamics by the proper choice of feedback although it will not be exponentially stable. One can also reduce the number of moduli by considering weaker forms of equivalence than smooth change of coordinates and invertible smooth feedback. We now discuss some important bifurcations of control sustems. Some of this has already been established by Kang.

\subsection{Fold Control Bifurcation}

Just as with classical bifurctions, the simplest control bifurcation is the fold. This control bifurcation has not been studied before. The uncontrollable part is one dimensional and unstable, $n_{1}=1, A_{1}>0$. Because the linearly controllable part of the quadratic normal form (2.6) is in Brunovsky form, the equilibria $z_{e}, v_{e}$ are most conveniently parametrized by $\mu=z_{e 2,1}$. The equilibria $z_{e}(\mu), v_{e}(\mu)$ are given by

$$
\begin{aligned}
& z_{e 1,1}=-\mu^{2} A_{1}^{-1} \delta_{1}^{11}+O(\mu)^{3} \\
& z_{e 2,1}=\mu \\
& z_{\epsilon 2, i}=O(\mu)^{2} \quad i=2, \ldots, n_{2}+1 .
\end{aligned}
$$

The local linearization around $z_{e}, v_{e}$ is

$$
\begin{aligned}
{\left[\begin{array}{c}
\dot{\tilde{z}}_{1} \\
\dot{z}_{2}
\end{array}\right]=} & \left(\left[\begin{array}{cc}
A_{1}+\mu \gamma_{1}^{11} & \mu \Delta \\
0 & A_{2}
\end{array}\right]+O(\mu)^{2}\right)\left[\begin{array}{c}
\tilde{z}_{1} \\
\tilde{z}_{2}
\end{array}\right] \\
& +\left(\left[\begin{array}{c}
0 \\
B_{2}
\end{array}\right]+O(\mu)^{2}\right) \tilde{v}
\end{aligned}
$$

where $\tilde{z}=z-z_{e}(\mu), \tilde{v}=v-v_{e}(\mu)$ and $\Delta=$ $\left[\begin{array}{llll}2 \delta_{1}^{11} & 0 & \ldots & 0\end{array}\right]$.

If the transversality condition $\delta_{1}^{11} \neq 0$ is satisfied then the system is linearly controllable hence stabilizable about any equilibrium except $\mu=0$. Consider a parametrized family of feedbacks $v=\kappa(z, \mu)$ of the form

$$
\tilde{v}=K_{1}(\mu) \tilde{z_{1}}+K_{2}(\mu) \tilde{z_{2}}
$$

Ideally one would like to find a continuous family of feedbacks that makes the family of equilibria asymptotically stable, i. e., for each small $\mu$, the closed loop systein $\dot{\tilde{z}}=\tilde{f}(z, \kappa(z, \mu))$ is asymptotically stable to $z_{e}(\mu)$. The lowest degree terms of more general smooth feedbacks will be like (3.18).
Clearly the the $z_{2}$ subsystem is stabilizable for all $\mu$ by proper choice of $K_{2}$ and this gain can be chosen independent of $\mu$. The question is can we find $K_{1}(\mu)$ which stabilizes the $z_{1}$ coordinate for all small $|\mu|$.

Since the linear approximations are stabilizable for $\mu \neq 0$, it is certainly possible to find a stabilizing feedback at each such $\mu$. The linear approximation at $\mu=0$ has an uncontrollable, unstable mode so it is not possible to stabilize it. But is it possible to stabilize the approximations for $\mu \neq 0$ with a feedback that is bounded through $\mu=0$ ? The answer is no for systems with a fold control bifurcation. For any bounded feedback, the closed loop system will be unstable in some neighborhood of $\mu=0$.

The closed loop linear approximation

$$
\left[\begin{array}{c}
\dot{\tilde{z}}_{1} \\
\tilde{\tilde{z}}_{2}
\end{array}\right]=\left(\left[\begin{array}{cc}
A_{1}+\mu \gamma_{1}^{11} & \mu \Delta \\
B_{2} K_{1} & A_{2}+B_{2} K_{2}
\end{array}\right]+O(\mu)^{2}\right)\left[\begin{array}{c}
\tilde{z}_{1} \\
\tilde{z}_{2}
\end{array}\right]
$$

is clearly unstable at $\mu=0$ since $A_{1}>0$. Furthermore if the feedback $v=K_{2}(\mu) z_{2}$ stabilizes the $z_{2}$ subsystem then $A_{1}$ is a simple positive root of the characteristic polynomial of the closed loop system when $\mu=0$. Hence there is a positive root of the characteristic polynomial for small $|\mu|$.

By using higher and higher gain, it is possible to stabilize the system closer and closer to $\mu=0$. But if the feedback (3.18) is continuous, at best it will stabilize only some small but not too small $\mu>0$ or only some small but not too small $\mu<0$. If a smooth family of feedbacks does stabilize the system for some small $\mu>0$, the parametrized closed loop system generically undergoes a classical fold bifurcation (also called a saddle-node bifurcation) at some smaller $\mu>0$.

We illustrate this with a simple example in normal form

$$
\begin{aligned}
& \dot{z}_{1}=z_{1}-z_{2}^{2} \\
& \dot{z}_{2}=v .
\end{aligned}
$$

The equilibria are $z_{e, 1}=\mu^{2}, z_{e, 2}=\mu, v_{\epsilon}=0$. Under the feedback $v=K_{1}(\mu) \tilde{z}_{1}+K_{2}(\mu) \tilde{z}_{2}$, the closed loop linear approximation is

$$
\left[\begin{array}{c}
\dot{\tilde{z}}_{1} \\
\dot{\tilde{z}}_{2}
\end{array}\right]=\left[\begin{array}{cc}
1 & -2 \mu \\
K_{1}(\mu) & K_{2}(\mu)
\end{array}\right]\left[\begin{array}{l}
\tilde{z}_{1} \\
\tilde{z}_{2}
\end{array}\right]
$$

where $\tilde{z}=z-z_{e}(\mu), \tilde{v}=v-v_{e}(\mu)$. This is asymptotically stable iff the trace is negative and the determinant is positive, which yields the inequalities $-2 \mu K_{1}(\mu)<$ $K_{2}(\mu)<-1$. Clearly there is no bounded $K_{1}(\mu)$ that satisfies these for all small $|\mu|$.

If we choose $K_{1}=10$ and $K_{2}=-2$ then the closed loop linear approximation is stable for $\mu>0.1$ and unstable for $\mu<0.1$. It undergoes a fold bifurcation at $\mu=0.1$. If we choose $K_{1}=-10$ and $K_{2}=-2$ then the closed 
loop linear approximation is stable for $\mu<-0.1$ and unstable for $\mu>-0.1$. It undergoes a fold bifurcation at $\mu=-0.1$.

To see this, consider the closed loop nonlinear system under the feedback with $K_{1}=10$ and $K_{2}=-2$ in coordinates centered at the the bifurcation, $\bar{z}_{1}=z_{1}-$ $0.01 . \bar{z}_{2}=z_{2}-0.1, \bar{\mu}=\mu-0.1$,

$$
\begin{aligned}
& \dot{\bar{z}}_{1}=\bar{z}_{1}-0.2 \bar{z}_{2}-\bar{z}_{2}^{2} \\
& \dot{\bar{z}}_{2}=10 \bar{z}_{1}-2 \bar{z}_{2}-10 \bar{\mu}^{2} .
\end{aligned}
$$

It is convenient to reparametrize by $\nu=\bar{\mu}^{2} \geq 0$. The center manifold is given by

$\bar{z}_{2}=10 \nu+5 \bar{z}_{1}+2500 \nu^{2}-1000 \nu \bar{z}_{1}+125 \bar{z}_{1}^{2}+O\left(\bar{z}_{1}, \nu\right)^{3}$

and the center manifold dynamics is

$$
\dot{\bar{z}}_{1}=2 \nu-600 \nu^{2}+300 \nu \bar{z}_{1}-500 \bar{z}_{1}^{2}+O\left(\bar{z}_{1}, \nu\right)^{3}
$$

or in the variables $\hat{z}_{1}=\sqrt{50}\left(\bar{z}_{1}-3 \nu\right), \hat{\nu}=2 \nu-150 \nu^{2}$,

$$
\dot{\hat{z}}_{1}=\hat{\nu}-\hat{z}_{1}^{2}+O\left(\hat{z}_{1}, \hat{\nu}\right)^{3}
$$

the familiar form of a fold bifurcation.

\subsection{Transcritical Control Bifurcation}

Kang [5] studied this control bifurcation but not under this name. This is the degenerate case of the above where again $n_{1}=1$ but $A_{1}=0$. Hence this control bifurcation is not generic. Kang showed that the local behaviour is determined by the roots of quadratic form

$$
\beta_{1}^{11} z_{1,1}^{2}+\gamma_{1}^{11} z_{1,1} z_{2,1}+\delta_{1}^{11} z_{2,1}^{2}=0
$$

If this form is positive or negative definite, then there is only an isolated equilibrium which is unstabilizable. If it is indefinite but not degenerate, there are two curves of equilibria which cross. For example, suppose $\beta_{1}^{11}=$ 1. $\gamma_{1}^{11}=0, \delta_{1}^{11}=-1$ and all the other nonlinear terms are zero. Then the equilibria are

$$
\begin{aligned}
& z_{e 1.1}^{ \pm}= \pm \mu \\
& z_{e 2.1}=\mu \\
& z_{e 2,4}=0 \quad i=2, \ldots, n_{2}+1 \text {. }
\end{aligned}
$$

Let $z_{e}(\mu), v_{e}(\mu)$ be one smooth curve of equilibria, e.g., $z_{c 1}^{+}$, the local linearizations around it are

$$
\begin{aligned}
{\left[\begin{array}{c}
\dot{\tilde{z}}_{1} \\
\dot{\tilde{z}}_{2}
\end{array}\right]=} & \left(\left[\begin{array}{cc}
2 \mu & \mu \Delta \\
0 & A_{2}
\end{array}\right]+O(\mu)^{2}\right)\left[\begin{array}{l}
\tilde{z}_{1} \\
\tilde{z}_{2}
\end{array}\right] \\
& +\left(\left[\begin{array}{c}
0 \\
B_{2}
\end{array}\right]+O(\mu)^{2}\right) \tilde{v}
\end{aligned}
$$

where $z=z-z_{e}(\mu), \tilde{v}=v-v_{e}(\mu)$ and $\Delta=\left[\begin{array}{lll}-2 & 0 \ldots\end{array}\right]$.

The linear approximation of the closed loop dynamics under a smooth feedback (3.18) is

$$
\left[\begin{array}{c}
\dot{\tilde{z}}_{1} \\
\tilde{\tilde{z}}_{2}
\end{array}\right]=\left(\left[\begin{array}{cc}
\mu & \mu \Delta \\
B_{2} K_{1} & A_{2}+B_{2} K_{2}
\end{array}\right]+O(\mu)^{2}\right)\left[\begin{array}{c}
\tilde{z}_{1} \\
\tilde{z}_{2}
\end{array}\right]
$$

which is neutrally stable at $\mu=0$. If the feedback stabilizes the $\tilde{z}_{2}$ subsystem when $\mu=0$ then the determinant of the full system matrix changes sign at $\mu=0$ so generically the closed loop system undergoes a fold bifurcation. Under quadratic feedback $v=K_{1}\left(z_{1}^{2}-\mu^{2}\right)+K_{2} z_{2}$ the system will undergo a transcritical bifurcation if $A_{2}+B_{2} K_{2}$ is Hurwitz.

\subsection{Hopf Control Bifurcation}

This control bifurcation has not been studied before. The uncontrollable modes are a nonzero complex conjugate pair,

$$
A_{1}=\left[\begin{array}{ll}
\lambda & 0 \\
0 & \bar{\lambda}
\end{array}\right]
$$

where $\lambda=\nu+i \omega, \bar{\lambda}=\nu-i \omega, \omega \neq 0$. The equilibria $z_{e}(\mu), v_{e}(\mu)$ are given by

$$
\begin{aligned}
{\left[\begin{array}{l}
z_{e 1,1} \\
z_{e 1,2}
\end{array}\right] } & =-\mu^{2} A_{1}^{-1}\left[\begin{array}{l}
\delta_{1}^{11} \\
\delta_{2}^{11}
\end{array}\right]+O(\mu)^{3} \\
z_{e 2,1} & =\mu \\
z_{e 2, i} & =O(\mu)^{2} \quad i=2, \ldots, n_{2}+1
\end{aligned}
$$

where $\delta_{1}^{11}, \delta_{2}^{11}$ is a complex conjugate pair.

The local linearization around $z_{e}, v_{e}$ is

$$
\begin{aligned}
{\left[\begin{array}{c}
\dot{\tilde{z}}_{1} \\
\dot{\tilde{z}}_{2}
\end{array}\right]=} & \left(\left[\begin{array}{cc}
A_{1}+\mu \Gamma & \mu \Delta \\
0 & A_{2}
\end{array}\right]+O(\mu)^{2}\right)\left[\begin{array}{c}
\tilde{z}_{1} \\
\tilde{z}_{2}
\end{array}\right] \\
& +\left(\left[\begin{array}{c}
0 \\
B_{2}
\end{array}\right]+O(\mu)^{2}\right) \tilde{v}
\end{aligned}
$$

where $\tilde{z}=z-z_{e}(\mu), \tilde{v}=v-v_{e}(\mu)$ and

$$
\Gamma=\left[\begin{array}{cc}
\gamma_{1}^{11} & \gamma_{1}^{21} \\
\gamma_{2}^{11} & \gamma_{2}^{21}
\end{array}\right], \quad \Delta=\left[\begin{array}{cccc}
2 \delta_{1}^{11} & 0 & \ldots & 0 \\
2 \delta_{2}^{11} & 0 & \ldots & 0
\end{array}\right]
$$

where $\gamma_{1}^{11}, \gamma_{2}^{11}$ and $\gamma_{1}^{21}, \gamma_{2}^{21}$ are complex conjugate pairs.

If the transversality condition $\Delta \neq 0$, is satisfied then the system is linearly controllable except when $\mu=0$. If $\nu<0$ then the system is stabilizable about any equilibrium but if $\nu \geq 0$ then the system is not stabilizable when $\mu=0$. The case $\nu \geq 0$ is called a Hopf control bifurcation. We distinguish two subcases, $\nu>0$ and $\nu=0$.

If $\nu>0$ then it requires larger and larger gain to stabilize the system closer and closer to $\mu=0$. But if the feedback (3.18) is continuous it will stabilize only for some small $\mu>0$ or for some small $\mu<0$ but not both. At $\mu=0$ the poles of the closed loop system are $\lambda, \bar{\lambda}$ and the poles of $A_{2}+B_{2} K_{2}(0)$. The latter can be made stable but the former are unstable. If the feedback is bounded then as $\mu \rightarrow 0$ the poles coverge to these. The system is controllable for $\mu \neq 0$ so the poles can be placed arbitrarily by feedback. The poles associated primarily with the $z_{2}$ subsystem can be kept 
stable but the two poles associated primarily with the $\therefore$ subsystem will cross into the right half plane at some small value(s) of $\mu$. Depending on the choice of feedback, they will cross one at a time as real poles, cross together through 0 or cross together as a nonzero complex conjugate pair. If they cross separately as real poles then generically the closed loop system undergoes a fold bifurcation as the first pole crosses. If they cross together as a nonzero complex conjugate pair then generically the system undergoes a Hopf bifurcation. If they cross together through zero the situation can be quite complicated and will not be discussed here.

If $\nu=0$ and the feedback (3.18) is continuous then generically the system undergoes a Hopf bifurcation at $\mu=0$. We illustrate this with an example.

$$
\begin{aligned}
\dot{x}_{1,1} & =-x_{1,2}-x_{2}^{2} \\
\dot{x}_{1,2} & =x_{1,1}-x_{2}^{2} \\
\dot{x}_{2} & =u .
\end{aligned}
$$

The equilibria are

$$
\begin{aligned}
x_{e 1,1} & =\mu^{2} \\
x_{e 1,2} & =-\mu^{2} \\
x_{e 2} & =\mu \\
u_{e} & =0
\end{aligned}
$$

and the linear approximations are

$$
\begin{aligned}
\dot{\tilde{x}}_{1,1} & =-\tilde{x}_{1,2}-2 \mu \tilde{x}_{2} \\
\dot{\tilde{x}}_{1,2} & =\tilde{x}_{1,1}-2 \mu \tilde{x}_{2} \\
\dot{\tilde{x}}_{2} & =\tilde{u}
\end{aligned}
$$

where $\tilde{x}_{1.1}=x_{1,1}-\mu^{2}, \tilde{x}_{1,2}=x_{1,2}+\mu^{2}, \tilde{x}_{2}=x_{2}-$ $\mu . \bar{u}=u$. The linear approximations are controllable except at $\mu=0$.

We choose the feedback $u=10 \tilde{x}_{1,1}+5 \tilde{x}_{1,2}-3 \tilde{x}_{2}$ which place the poles at $-1 \pm i,-1$ when $\mu=0.1$. Near $\mu=0$ the poles of the closed loop linear approximations are

$$
\begin{aligned}
& s=-3+10 \mu+O(\mu)^{2} \\
& s=-5 \mu \pm i+O(\mu)^{2} .
\end{aligned}
$$

The closed loop dynamics is

$$
\begin{aligned}
\dot{x}_{1,1} & =-x_{1,2}-x_{2}^{2} \\
\dot{x}_{1,2} & =x_{1,1}-x_{2}^{2} \\
\dot{x}_{2} & =10 x_{1,1}+5 x_{1,2}-3 x_{2}+3 \mu-5 \mu^{2} .
\end{aligned}
$$

The first Lyapunov coefficient (see [8], (10.50)) is $l_{1}(0)=-70.11$. This and (3.19) imply that the closed loop system undergoes a supercritical Hopf bifurction at $\mu=0$. For small $\mu>0$ the origin is locally exponentially stable. For small $\mu<0$ the origin is unstable but there is a locally asymptotically stable limit cycle nearby. At $\mu=0$ the origin is locally asymptotically stable but not locally exponentially stable.

\section{Conclusions}

We have presented the normal form to degree 3 of a smooth control system around an equilibrium point under the group of smooth coordinate changes and invertible smooth feedback. From these normal forms we were able to identify the simplest control bifurcations, the fold control bifurcation, the transcritcal control bifurcation and the Hopf control bifurcation. We emphasize the distinction between a control bifurcation and a classical bifurcation. A control bifurcation occurs at an equilibrium where the control system loses linear stabilizability. A classical bifurcation occurs at an equilibrium where the dynamical system loses structural stability with respect to parameter variations. Bifurcation control is different from a control bifurcation. The former refers to the modification by feedback of a classical bifurcation of the $u=0$ system. Of course these concepts are closely related and when a system with a control bifurcation is modified by smooth feedback the result is a classical bifurcation.

\section{References}

[1] E. H. Abed and J.-H. Fu, Local Feedback Stabilization and Bifurcation Control, Part I. Hopf Bifurcations, Systems and Control Letters, 7 (1986), 11-17.

[2] E. H. Abed and J.-H. Fu, Local Feedback Stabilization and Bifurcation Control, Part II. Stationary Bifurcations, Systems and Control Letters, 87 (1987), 467-473.

[3] J.-P. Barbot, S. Monaco and D. Normand-Cyrot, Quadratic forms and feedback linearization in discrete time, Int. J. Control, 67 (1997), 567-586.

[4] W. Kang and A. J. Krener, Extended quadratic controller normal form and dynamic feedback linearization of nonlinear systems, SIAM J. Control and Optimization, 30 (1992), 1319-1337.

[5] W. Kang, Bifurcation and normal form of nonlinear control systems - part I and part II, SIAM J. Control and Optimization, 36 (1998), 193-212 and 213232.

[6] A. J. Krener, W. Kang and D.-E. Chang, Control Bifurcations, in preparation.

[7] L. Li and A. J. Krener, Quadratic and Cubic Normal Forms of Discrete Time Nonlinear Control Systems, Anais do XIII Congresso Brasileiro de Automatica, CBA 2000, pp. 19-25.

[8] Y. A. Kuznetsov, Elements of Applied Bifurcation Theory. Springer-Verlag, New York, 1998. 